\title{
LA UTILIZACIÓN DEL VÍDEO PARA LA ENSEÑANZA DE CONCEPTOS BÁSICOS (CALOR Y TEMPERATURA)
}

\author{
INSAUSTI, M.J. ${ }^{1}$, BELTRÁN, M.T. ${ }^{2}$, CRESPO, M.S. ${ }^{2}$ y GARCÍA, R. ${ }^{3}$ \\ i Escuela Universitaria Formación del Profesorado de EGB. Universidad de Valladolid. \\ 2 IB Antonio Tovar (Valladolid). \\ 3 IFP Galileo Galilei (Valladolid).
}

\section{SUMMARY}

The goal of one of the inquiries implemented in the ANTEC Project (Aplicación de Ias Nuevas Tecnologías a la Enseñanza de las Ciencias) was to analyze the use of vídeotapes as a means to teach Sciences. In this paper we present the use of vídeotapes related to Heat and Temperature Units, in classrooms with students who were 16 years old. We expected to modify their concepts about these subjects. The results suggest the improvement of students' concepts and vocabulary, although it was not the same with the application of scientific knowledge to everyday events.

\section{INTRODUCCIÓN}

La aparición de Ias NTI (Nuevas Tecnologías de la Información) ha propiciado un cambio social a gran escala. Cambio que conduce hacia una notable diversificación de las herramientas utilizables por el enseñante para desempeñar su labor (Bartolomé 1989), y cambio que implica una necesaria y profunda modificación curricular orientada al desarrolilo de habilidades que faciliten al alumno la adaptacion a un mundo altamente tecnologizado.

En la conferencia internacional sobre «Tecnología y educación del profesorado» celebrada en Monterrey (California) en agosto de 1989, se han puesto de manifiesto cuatro tipos de problemas que afectan al profesorado en esta situación de cambio (Marco y Linn 1989):

EI primero de estos problemas surge cuando el profesor desconoce la herramienta que la tecnología pone a su disposición. El segundo problema surge de la fuerte resistencia al cambio por parte del profesorado. EI tercer problema se refiere a la integración de la nueva tecnología en las actividades escolares. El cuarto problema se debe a la falta de nuevos métodos de valoración del aprendizaje adecuados a las modificaciones que las nuevas tecnologías introducen en el proceso de aprendizaje.
Lograr una solución para estos problemas seguramente no va a ser una tarea fácil.

En España, el Ministerio de Educación y Ciencia puso en marcha en 1984 el proyecto ATENEA con la intención de investigar Ias posibles aplicaciones de Jos ordenadores en la enseñanza general básica y en las enseñanzas medias. En 1985, comenzó a desarrollar el proyecto MERCURIO con la intención de analizar los usos del vídeo en el proceso de enseñanza-aprendizaje. Se han invertido grandes sumas de dinero en la formación de monitores especializados en el uso del vídeo y del ordenador como herramienta educativa, así como en la dotacion de hardware y software a los centros de EGB y enseñanzas medias. Pero el profesorado, en su mayoria, aún no ha integrado estas herramientas en el desarrollo deI currículo y, por otra parte, la dotación de material todavía no es suficiente.

Éste es el contexto en el que abordamos este trabajo. Ha sido desarrollado dentro deI proyecto ANTEC (Aplicación de las Nuevas Tecnologías a la Enseñanza de las Ciencias), proyecto propiciado mediante un convenio entre las universidades de Madrid, Valladolid y el Programa de Nuevas Tecnologías del Ministerio de Educación y Ciencia (1990). 
EI objetivo que pretendíamos era conseguir la integración de las NTI (en este caso el vídeo) en la práctica diaria đel profesor para que los alumnos interrelacionen e integren distintas fuentes de información y métodos de trabajo.

Uno de los aspectos que intentamos investigar fue la aportación del vídeo en el proceso de enseñanza-aprendizaje en to que respecta a:

a) La mejora del lenguaje científico utilizado por los alumnos. Inconscientemente el profesor en su práctica docente diaria puede estar utilizando un lenguaje científico que adolezca de la precisión necesaria.

b) La adquisición de conceptos científicos. Como dicen Viennot (1985) y Driver (1986), los estudiantes están ya en posesión de sus propios esquemas conceptuales at respecto, elaborados en un intento de explicación racional de sus experiencias cotidianas previas, y son muy reacios al cambio conceptual. ¿Puede ayudar el vídeo a que los alumnos modifiquen estos conceptos espontáneos hacia sus correspondientes conceptos científicos?

\section{DESARROLLO DE LA INVESTIGACIÓN}

Este trabajo ha sido llevado a cabo con alumnos de 16 años en dos centros diferentes: Instituto de Bachillerato Antonio Tovar, e Instituto de Formación Profesional Galileo Galilei. El primer centro está bien situado en la ciudad y los 38 alumnos del aula en la que se ilevó a cabo este trabajo proceden de un nivel social medio. Los 34 alumnos del segundo centro, que está ubicado en las afueras de la ciudad, proceden de un nivel social bajo.

Los temas en los que se utilizaron los vídeos estaban relacionados con el calor y la temperatura. En concreto:

1) Diferencia precisa entre energía térmica, calor y temperatura.

2) Cambios de estado.

Hemos elegido estos temas con objeto de estudiar el apoyo que podían prestar Ios vídeos para el aprendizaje de conceptos complejos y frecuentemente mal entendidos. De hecho, en la década de los af́os setenta se plantearon discusiones en torno a la conveniencia o no de introducir conceptos de termodinámica en los currículos de enseñanza primaria y secundaria. En el estudio realizado por Cervantes (1987) encontraremos una revisión bibliográfica sobre los mismos.

El material de vídeo que se utilizó después đe revisar el que existía en el mercado fueron tres de las ocho cintas de ia editoria Didascalia acerca del tema "Calor y Temperatura: Efectos deI CaIor».

Los profesores analizaron los vídeos y su utilidad didáctica, mediante la Guía de Análisis elaborada por el equipo del proyecto ANTEC de la Universidad de Valla- dolid. Esta guía se basa en un sistema de preguntas cerradas (202 en total) acerca del material y su uso en el aula, de las que puede verse un ejemplo en el anexo I. Las listas de chequeo se utilizan con frecuencia como herramienta para lievar a cabo la valoración de productos destinados al uso didáctico. Podemos encontrar listas de este tipo en los trabajos de Croft y Evans (1985), Chamber y Sprecher (1983) o Blease (1986) donde se debate su idoneidad en los procesos de valoración del software informático utilizado en las escuelas. También de forma frecuente, encontramos Iistas de chequeo en las revistas de información general sobre informática o vídeo y en algunos manuales sobre valoración de materiales curriculares dirigidos aI profesorado (Curriculum Evaluation and Assessment in Educational Institutions, 1981). Las listas de chequeo presentan las siguientes ventajas: proporcionan un esquema de trabajo sistemático para hacer una valoración, asegurando a su usuario que no ha olvidado ningún punto importante. Son particularmente útiles en la selección de material fuera del aula, son fáciles de manejar y su aplicación es relativamente rápida.

Pues bien, con las respuestas dadas por el profesor a la Guía podrá juzgar él mismo la calidad del vídeo en términos de enseñanza-aprendizaje. Se pretendía facilitar con esta herramienta la reflexión analítica sobre la calidad educativa de un recurso didáctico (Sigüenza Molina y Sáez 1990)

Para el análisis de la comprensión de los temas por parte de los alumnos, se elaboró un test de 15 preguntas de formulación cerrada (anexo II). El material bibliográfico en el que nos basamos para la confección de estos tests se recoge en las referencias bibliográficas que cierran el artículo.

El proceso seguido para la realización đe la experiencia llevada a cabo ha sido el siguiente:

\section{Instituto de Bachillerato Antonio Tovar}

1. Explicación teórica del tema por parte del profesor, con realización de ejercicios prácticos.

2. Aplicación del test elaborado.

3. Proyección de los vídeos en el aula.

4. Repetición del test.

Instituto de Formación Profesional Galileo Galilei

1. Aplicación del test.

2. Proyección de los vídeos en el aula.

3. Nueva aplicación del test.

4. Explicación teórica por parte del profesor.

5. Prueba final elaborada con preguntas diferentes a las del test, pero haciendo hincapié en los mismos conceptos. 


\section{RESULTADOS}

En este apartado vamos a reflejar sólo el trabajo realizado en el IB Antonio Tovar pues, aunque los procesos hayan sido distintos, las conclusiones a las que Ilegamos en ambos centros son similares.

Las 15 cuestiones del test se pueden agrupar en cuatro categorías:

a) Conceptuales $(1,2,3,4,5)$.

b) Relación de conceptos entre sí $(6,7,8,9)$.

c) Aplicación de conceptos para explicar hechos de la vida real $(10,11,12,13)$.

d) Relación de este tema con conceptos adquiridos anteriormente $(14,15)$.

Figura 1

Respuestas antes de proyectar el vídeo.

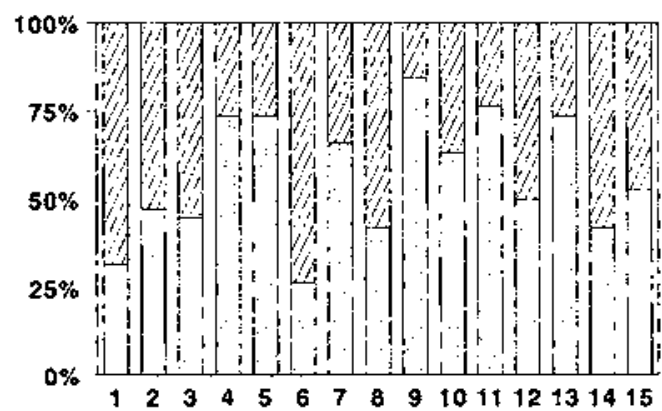

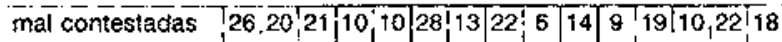

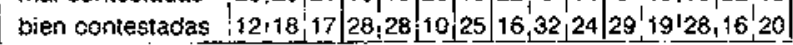

Num. de preguntas

○bien contestadas $Z_{2}$ mal contestadas

Los tests siempre fueron analizados clasificando las respuestas de los alumnos como bien o mal, pero nunca de forma intermedia o mediante notas. Los resultados deI análisis de los tests los podemos ver en las figuras I y 2. En la figura 3 vemos una comparación de las respuestas en lo que respecta a un mejoramiento de las contestaciones o a un empeoramiento a pesar de haber insistido en los conceptos pasando los vídeos.

Un análisis detallado nos indica que en general el aumento más espectacular está en las preguntas de concepto: los alumnos mejoran mucho en cuanto al léxico utilizado y a la interpretación del concepto. En las preguntas de aplicación del método cientifico a los hechos de la vida cotidiana es donde existe mayor dificultad de aprendizaje.

Las preguntas relacionadas con los conceptos de energía térmica, calor y temperatura, que están muy bien expuestas y visualizadas en el vídeo, tienen unas mejoras considerables.
Sin embargo, en conceptos y hechos experimentales como el mantenimiento de la temperatura en los cambios físicos, que también está muy bien expresado en el vídeo, no se han dado los resultados esperados.

Figura 2

Respuestas después de proyectar el vídeo.

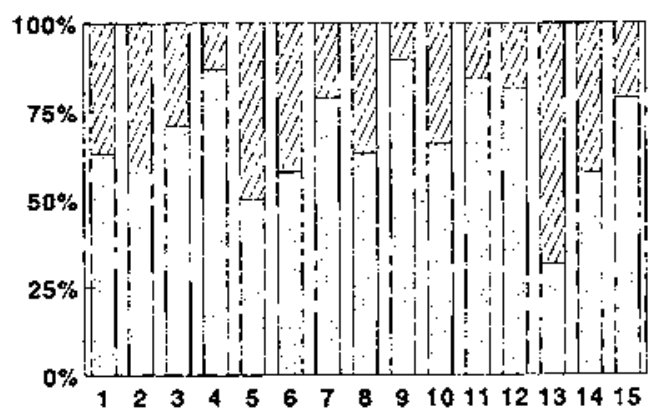

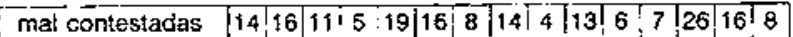

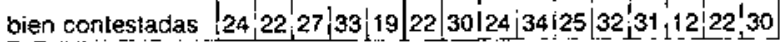

Núm. de preguntas

- bien contestadas $7<$ mal contestadas

Figura 3

Comparación de respuetas.

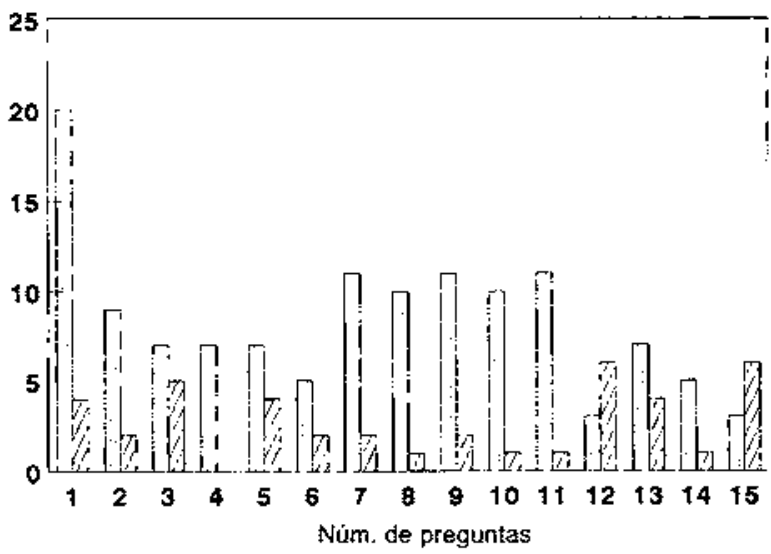

[]$_{\text {mejor }}$ contestadas $[/ / 2$ peor contestadas

\section{CONCLUSIÓN}

1) La utilización de este recurso audiovisual ha servido para mejorar el léxico de los alumnos en la expresión escrita de los conceptos aprendidos.

2) También ha aumentado el grado de comprensión por parte de los alumnos de los conceptos expuestos de forma directa en el vídeo. 
3) Sin embargo, el fin último del aprendizaje de las ciencias, que es la comprensión racional y razonada de

\section{REFERENCIAS BIBLIOGRÁFICAS}

BARTOLOMÉ, A., 1989. Nuevas tecnologías y enseñanza. (Grao: Barcelona).

BLEASE, D., Evaluating educational software. (Ed. Billing \& Sons limited: Worcester, Gran Bretaña).

CERVANTES, A, 1987. Los conceptos de calor y temperatura: Una revisión bibliográfica, Enseñanza de las Ciencias, Vol. 5, pp. 66-70.

CROFT, G. y EVANS, S., 1985. Educational Software Review Ptoject, Computer Education.

Curriculum Evaluation and Assessment in Educational Institutions, 1981. (Ed. Open University Press, Mitton Keynes: Londres, Gran Bretaña).

CHAMBER, J.A. y SPRECHER, J.W.,1983. Computer Assisted Instruccion. Its Use in the Classroom. (Ed. Prentice Hall: EEUU).

DRIVER, E., 1986. Psicología congnoscitiva y esquemas conceptuales en Ios alumnos, Enseñanza de las Ciencias, Vol. 4, pp. 3-15.

MARCO, R. y LINN, M., 1989. Tecnología e instrucción: promesa y problemática, Revista de Educacion, 288, pp. $391-403$

SIGÜENZA MOLINA, J. F. y SÁEZ BREZNES M.J., 1990. Actas VII Jornadas de Estudio sobre la Investigación en la Escuela. Sevilla

VIENNOT, L., 1985. Analysing students ' reasoning in science: A pragmatic view of theoretical problems, European Journal of Science Education, Vol. 7, pp. 151-162. los hechos cotidianos a través de las leyes físicas, no se ha alcanzado en grado satisfactorio.

\section{BIBLIOGRAFÍA SOBRE EL CALOR}

ALONSO, M. y FINN E.J., 1970. Física, Vol. III. (Fondo Educativo Interamericano: México).

BERKELEY, 1969. Física Estadística. (Ed. Reverté: Barcelona).

FERNÁNDEZ URIA, E., 1986. Reflexiones acerca del concepto de calor, Enseñanza de las Ciencias, Vol. 4, pp. $91-92$.

GARCÍA HOURCADE, J.L. y RODRIGUEZ DE ÁVILA, C. 1985. Preconcepciones sobre el calor en $2^{\circ}$ de BUP, Enseñanza de las Ciencias, Vol, 3, pp. 188-193.

GUESNE, E., TIBERGHIEN, A. y DELACOTE G., 1978. Méthodes et resultats concernat l'analyse des conceptions des éleves dans différents domaines de la phisique- Deux examples: les notions de chaleur et lumière, Révue Francaise de Pédagogie, 45.

HIERREZUELO MORENO, J., MOLINA GONZÁLEZ, E. y MONTERO MORENO, A., 1987. Consideraciones sobre la enseñanza del calor en $2^{\circ}$ de BUP, Enseñanza de las Ciencias, Vol. 5, pp. 81-83.

HOLTON, G. y ROLLER, H.D., 1972. Fundamentos de Física Moderna. (Ed. Reverté: Barcelona).

MACEDO de BURGH1, B. y SOUSSAN, G., 1985. Estudio de los conocimientos preadquiridos sobre las nociones de calor y temperatura en alumnos de 10 a 15 años, Enseñanza de las Ciencias, Vol. 3, pp. 83-90.

NACHMIAS, R., STAVY, R. y AURAMS, R., 1990, A microcomputer-based diagnostic system for identifying students' conception of heat and temperature, International Journal of Science Education, Vol. (12) pp. 123-132.

SUMMERS, M.K., 1983. Teaching heat and analysis of misconceptions, The School Science Rewiew, Vol. 64, pp. 670-676.

TEJERINA, F., 1976. Termodinámica. (Paraninfo: Madrid).

VÁZQUEZ DÍAZ, J., 1987. Algunos aspectos a considerar en la dídáctica del calor, Enseñanza de las Ciencias, Vol. 5(3), pp. $235-238$. 


\section{ANEXO I}

Guía de análisis

(Incluímos algunas de las preguntas que constituyen la guía de análisis citada en el texto.)

2.4. Valoración del contenido.

a. ¿Los contenidos se ajustan plenamente al tema al que se hace referencia en el título del vídeo?

b. ¿Los contenidos se corresponden con el tema del currículo que se pretende estudiar?

c. ¿Los contenidos se adaptan a las características de nuestros alumnos?

d. ¿La veraciodad, rigurosidad, exactitud y precisión de la información es del todo adecuada?

1. ¿Algún concepto se utiliza con más de un significado?

e. ¿La integración del audio con la imagen es adecuada?

1. ¿Existe una buena sincronización entre el audio y la imagen?

2. ¿El volumen de información oral resulta adecuado para el volumen de información visual?

3. La locución es:

- ¿adecuada?

- ¿monótona?

- ¿demasiado expresiva?

f. EI vídeo puede ser adecuado para:

- motivar

- transmitir información

- enseñar una destreza

- aprender un concepto

- estimular un debate

- modificar actitudes

- estimular la imaginacíón

(Indique su opinión). 


\section{ANEXO II}

\section{TEST}

1. ¿A qué llamamos enegía térmica de las sustancias?

2. ¿Cómo entiendes el concepto de temperatura?

3. ¿A qué denominamos calor de fusión?

4. El calor y la temperatura:

- son lo mismo

- son dos formas diferentes de energía

- el primero es una energía y el segundo un efecto del calor

- el primero es uná energía çue pasá de un cuerpo a otro y la segunda mide la energía cinética media de las moléculas.

- no tiene nada que ver

5. ¿Es correcto decir que este cuerpo tiene mucho calor?

6. Diferencia los conceptos de fusión y solidificación. Explícalo

7. ¿Qué supones que tarda menos en elevar su temperatura, al calentarlos con un mechero, el aceite o el agua? ¿Por quée?

8. Dígase a quế temperatura las partículas de un cuerpo están teóricamente inmóviles.

9. ¿Qué hace falta para que pase energía térmica de un cuerpo a otro?

10. ¿Cómo es posible que calentando bielo éste se funda sin elevar la temperatura? ¿Qué ha pasado con el calor?

11. Como sabes, las vías del ferrocarril no se hacen continuas, sino que se dejan unos espacios entre cada tramo. ¿Por qué?

12. ¿Por qué en verano al pisar la arena de la playa puedes quemarte el pie, pero, si pisas el agua, no ocurre nada?:

- porqu la la arena tiene más calor y está, por tanto, más caliente

- porque el agua al recibir el mismo calor del sol aumenta menos su temperatura

- porque el agua está húmeda y quita parte del calor al sol

- porque la temperatura que alcanzan los líquidos es siempre menor que la que alcanzan los sólidos.

13. ¿Por qué en las montañas el agua hierve a menos temperatura?

14. La densidad de los cuerpos, en general, disminuye al aumentar la temperatura. ¿Por qué?

15. Si un día que vas de viaje mides la presión de las ruedas del coche antes de arrancar, observarás que es menor que si ta mides después de recorrer muchos kilómetros. ¿̨Por qué? 